\title{
Role of Magnetic Resonance Angiography in Evaluation of Brain Pathologies
}

\author{
Varkala Srinivas ${ }^{1}$, Sai Venkata Rammohan ${ }^{2}$
}

\author{
1. Assistant Professor, Department of Radiology, Prathima Institute of Medical Sciences, Naganoor, \\ Karimnagar.
}

2. Professor, Department of Radiology, Prathima Institute of Medical Sciences, Naganoor, Karimnagar.

Address for Correspondence:Dr. Sai Venkata Rammohan, Professor, Department of Radiology, Prathima Institute of Medical Sciences, Naganoor, Karimnagar. Email:vcnu99@gmail.com

Date of Receiving: 6/03/2021

Date of peer review: 13/03/2021

Date of Acceptance: 19/03/2021

\section{DOI:10.47799/pimr.0902.17}

\begin{abstract}
Background: MR angiography is the latest technique for the evaluation of cerebrovascular diseases. It is now used commonly for the evaluation of brain pathologies. The advantage is it is a non-invasive method of brain vasculature. The present study aimed to evaluate the role of Magnetic Resonance Angiography in the detection of brain pathologies and to study the Role of Magnetic Resonance Angiography in Cerebral Arteriovenous Malformations, Aneurysms, and Cerebral infarctions.
\end{abstract}

Methods: Data for the study was collected from patients with clinically suspected cerebral lesions or from patients, in whom previous images depicted cerebral lesions, undergoing MRA for evaluation of cerebral lesions Patients underwent MR imaging including TOF Magnetic resonance angiography according to the set protocols after obtaining informed consent. All these patients had undergone detailed clinical evaluation by the referring neurosurgery unit.

Results: In this study, a total of $n=32$ cases were included out of which $n=6(18.75 \%)$ were AVM, $n=10(31.25 \%)$ were Aneurysms, and $n=16(50 \%)$ were cases of infarcts. Of the total $n=16$ infarct cases $n=8(50 \%)$ were found in the middle cerebral artery (MCA) $n=4(25 \%)$ in ACOM and $n=2$ in PCOM, as well as Internal carotid artery (ICA). Of the MCA cases $n=5(62.5 \%)$ were right-sided and $n=3(37.5 \%)$ were left-sided.

Conclusion: MRA is now in routine use as a non-invasive tool for imaging the cerebralvasculature. In cerebrovascular disease, it is the investigation of choice forpatients who are suspected/ increased risk of having unruptured intracranial aneurysms, and intracranial vascular disease associated with acute infarction, intracranial dissection of the carotid and/or vertebral arteries, and follow up cases of Cerebral AVMs.
Keywords: Arteriovenous malformations, Magnetic resonance angiography, aneurysms, cerebral infarcts

\section{INTRODUCTION}

Magnetic Resonance Angiography is a rapidly evolving technique for non-invasive vascular imaging. Since 1985, when it was first shown to be clinically feasible, the imaging techniques and hardware used for MR angiography have greatly improved.[1]MRA has become an essential component of $\mathrm{MRI}$ in the evaluation of many types of cerebrovasculardiseases. In the cases of acute stroke, MRA is useful for determining theseverity of stenosis, vascular occlusion, and collateral flow. ${ }^{[2]} 3 \mathrm{D}$ TOF technique has relatively high sensitivity and specificity indifferentiating surgical from nonsurgical carotid stenoses. Three-dimensionalTOF MRA is quite sensitive and specific for the evaluation of intracranialproximal stenoses and occlusions. ${ }^{[3]}$ Twodimensional PC MRA is useful fordetermining collateral flow patterns in the circle of Willis.MRA is also useful inthe determination of stroke etiologies such as dissection, fibromusculardysplasia, vasculitis, and moya moya. ${ }^{[3]} \mid \mathrm{t}$ is now in routine use as a non-invasive tool for imaging the cerebralvasculature. In cerebrovascular disease, it is the investigation of choice forpatients who are suspected of having disorders such as unruptured intracranial aneurysms. Intracranial vascular diseases associated with acute cerebral infarctions. Transient ischemic attacks and intracranial or extracranial dissection of carotid and vertebral arteries. MRA can clearly define the circle of Willis sufficiently to allow detection of intracranialaneurysms as small as 3-4 mm. MRA holds promise as a truly non-invasiveintracranial vasculature screening examination in patients at risk foraneurysms. ${ }^{[4]}$ TOFMRA which provides information about vascular patency,caliber, has shown value in the diagnosis, localization, 
and follow-upof patients with moya moya disease and other intracranial vasculopathy.TOF \& PC techniques allow visualization of cerebral vasculature withoutadministration of contrast therefore it plays a vital role in patients who areallergic to contrast and in patients with renal failure in whom contrast iscontraindicated. ${ }^{[5]}$ Further advances in hardware and software in the future will result in improved resolution of smaller vessels. MRA is likely to replace angiographyfor a large number of clinical applications.The current study aims to determine the role of Magnetic Resonance Angiography in the evaluation ofbrain pathologies.

\section{Materials and Methods:}

This prospective study was conducted in the Department of Radiology \& imaging, Prathima Institute of Medical Sciences, Nagunoor. Karimnagar. Institutional ethical committee permission was obtained before the study. Written consent was obtained from all the participants of the study. Data for the study were collected from patients with clinically suspected cerebral lesions or from patients, in whom previous images depicted cerebral lesions, undergoing MRA for evaluation of cerebral lesions Patients underwent MR imaging including TOF Magnetic resonance angiography according to the set protocols after obtaining informed consent. All these patients had undergone detailed clinical evaluation by the referring neurosurgery unit.

\section{Inclusion Criteria}

1. Patients of age 12 years \& above with clinical/ CT suspicion of cerebralvascular malformations.

2. Patients already diagnosed of having cerebral AVM, Aneurysms, andInfarcts on CT scan or CT angiography or DSA and requiring MRImaging evaluation before radiosurgery/ embolization.

3. Follow up patients of cerebral AVM, for assessing the adequacy of thetreatment / residual AVM.

Exclusion Criteria

1. Patients were excluded if contraindications to MR imaging (claustrophobia,

2. pacemaker, potentially magnetic implants, etc) were present.

3. Patients requiring emergency vascular intervention.

4. Patients with severely impaired renal function, heart failure, and poor

5. physical conditions which precluded contrast administration.

\section{Patients who were pregnant.}

MR Imaging Protocol: MR imaging examinations were performed with a 16 channel $1.5 \mathrm{~T}$ whole-body imager (Achieva 1.5 Tesla, Philips Medical System, Netherlands.)equipped with a high-performance gradient system. A commercially available16 channel head and neck coil was used for imaging all the patients. Patientswere positioned supine on a scanner table and head immobilization wasachieved. First, routine MR imaging of the brain including T1WI, T2WI, and FLAIRsequences was done. Following this, 3D Multi Chunk Inflow Sequence wasused for Time of Flight angiography (TOF).Time of Flight MRA:3D Multi Chunk inflow sequence was used for TOFangiography. The scan was done by selecting a section thickness of 0.8- 1mm,over a 200-250-mm FOV (the upper thorax to the cranial vertex). Acquisitionparameters of the sequence as follows: TR/TE, 23/6.5 ms, flip angle, 20 degrees;Slice Orientation Transverse, with $20 \mathrm{~mm}$ thickness of single Saturation band,Image Matrix 512×512 with a scan duration of 5 minutes. Maximum intensityprojection (MIP) reconstruction was performed in-line.Post-processing and Image Analysis:After the acquisition of data, imagepostprocessing was performed on a 3D workstation (Philips Medical System), with standard commercial software by using the MIP algorithm. Overlapping thinMIP subvolumes $(10 \mathrm{~mm}$, with $9 \mathrm{~mm}$ overlap) in the coronal, sagittal, and axialplanes (or any desired obliquity) were reconstructed for all source images.Image Evaluation:Two neuroradiologists independently reviewed the TOFMR Angiography results using source and MIP images for image quality andCharacteristics of (Arteriovenous Malformation) AVM and Aneurysms.

\section{Results}

In this study a total of $n=32$ cases were included out of which $n=6(18.75 \%)$ were AVM, $n=10(31.25 \%)$ were Aneurysms, and $n=16(50 \%)$ were cases of infarcts. Out of the total $n=32$ cases, $n=18(56.25 \%)$ were males and $n=14(43.75 \%)$ were females. Among the Arteriovenous Malformations (AVM) cases $n=4$ $(66.67 \%)$ were males and $n=2(33.33 \%)$ were females male to female ratio of 2:1. $N=350 \%$ of AVM's were found in the Second Decade and 33.33\% seenin the third decade of life. In this study AVM's are most observed in Supratentorial location,only one AVM observed in infratentorial location. 33.33\% AVM's are observedin the Rightfrontal region, $16.66 \%$ observed in the Left Frontal region.16.66\% of AVM'sare seen in the Parietal region on each side.

Table 1: Distribution of AVM According to anatomical location

\begin{tabular}{|c|c|c|c|}
\hline Side & Frontal & Parietal & Infratentorial \\
\hline Right & $2(33.33 \%)$ & $1(16.66 \%)$ & $1(16.66 \%)$ \\
\hline Left & $1(16.66 \%)$ & $1(16.66 \%)$ & - \\
\hline
\end{tabular}


Of the total $n=10$ cases of Aneurysms were commonly seen in $n=4(40 \%)$ in $40-49$ years $n=3(30 \%)$ in $50-59$ years and $n=2(20 \%)$ in $60-69$ years and $n=1(10 \%)$ in $30-39$ years. In this study aneurysms are most seen in Acom followed by MCA - Middle Cerebral Artery(MCA) 40\% are seen in Anterior communicating Artery (Acom) and $30 \%$ are seen in MCA. In

\begin{tabular}{|c|c|c|}
\hline Location & Mean size $(\mathrm{mm})$ & Frequency of Aneurysms \\
\hline ACOM & 6.23 & $3(40 \%)$ \\
\hline MCA & 7.03 & $1(10 \%)$ \\
\hline PCOM & 6.1 & $1(10 \%)$ \\
\hline ICA (Cavernous) & 9 & 0 \\
\hline A1 & 0 & $1(10 \%)$ \\
\hline A2 & 5.4 & - \\
\hline Basilar Tip & - & $10(100 \%)$ \\
\hline Total & - & \\
\hline
\end{tabular}

According to this study, the total number of infracts cases was $n=16$. Males were $n=10$ and females were $n=6$ the male to female ratio was $1: 0.6$. The most common age group for infarct is 7 thDecade accounting for $37.5 \%$ of total infarcts followed by 6 th decade, $25 \%$ of Infarcts are observed in this age group depicted in figure 1.Infarcts are more commonly observed in males compared to females With, Male to female ratio of 1.63 .

Figure 1: Distribution of Infarcts According to Age

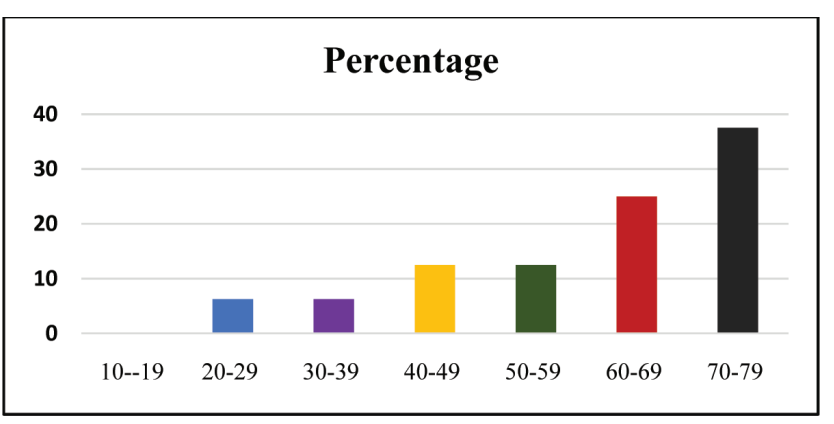

Of the total $n=16$ infarct cases $n=8(50 \%)$ were found in the middle cerebral artery (MCA) $n=4(25 \%)$ in ACOM and $n=2$ in PCOM, as well as Internal carotid artery (ICA). Of the MCA cases $n=5(62.5 \%)$ were right sided and $n=3(37.5 \%)$ were leftsided.

\section{Discussion}

In this study, Patients are having brain pathologies like AVM's,Aneurysms, and Infarcts.Among $n=32$ Patients, $n=6$ $(18.75 \%)$ patients had $A V M, n=10(31.25 \%)$ patients had
Acom, the mean size of the aneurysm is $6.23 \mathrm{~mm}$ and in MCA the mean size was $7.03 \mathrm{~mm}$. The most common size of the aneurysm is $6-7 \mathrm{~mm}$, accounting for $50 \%$ of Overallaneurysms. Among these $60 \%$ of aneurysms are observed in Acom (Table 2).

Table 2: Mean size and number of aneurysmsaccording to Location

Aneurysms and $16(50 \%)$ patients are havingCerebral Infarcts So, the majority of patients had cerebral infarcts in this study. Out of $n=32$ patients $n=18(56.25 \%)$ cases are male and $14(43.75 \%)$ cases arefemale patients.Arteriovenous malformations (AVMs) are defects of the circulatory systemthat are generally believed to arise during embryonic or fetal development orsoon after birth. ${ }^{[6,7]}$ They are composed of a complex tangle of arteries andveins connected by one or more fistulae. ${ }^{[6-8]}$ The pathogenesis ofarteriovenous malformations (AVMs) is not well understood.[8]According to literature the common age at presentation is between 20 and 40years. Most AVMs become symptomatic by 50 years of age. ${ }^{[9]}$ in this study, we had $n=4$ cases of AVM in males and $n=2$ in females. Similar findings have been reported by other studies [Meisel et al,] Headache with or without vomiting was the most common complaint $(93.3 \%)$ and seizures was the second common complaint $(60 \%)$ with which thepatients had presented to the referring unit. Other associated complaints likeloss of consciousness and weakness have also been described. ${ }^{[10]}$ Analysis of NCCT findings showed that intracranial hemorrhage wasthe most common finding and Intraventricular hemorrhage was also seen in somepatients. SAH was not seen in our study which is the most commonpresentation in cerebral aneurysms.In this study, all patients had a single AVM nidus which is usually a commonpresentation as described in other studies. AVM with Multiple nidi is a lesscommon presentation according to many studies. About $98 \%$ of AVMs areusually solitary. Although approximately $2 \%$ are multiple and they are usuallyassociated with extracerebral cutaneous or vascular anomalies. MultipleAVMs are seen in Rendu-Osler-Weber 
(ROW) and Wyburn-Masonsyndromes. ${ }^{[9]}$ Imaging that accurately defines the vascular anatomic featuresof AVMs is crucial for successful management. Yu S et al; ${ }^{[11]}$ Conducted a study to evaluate the clinical utility of anovel non-contrast fourdimensional (4D) dynamic MRA (dMRA) in theevaluation of intracranial AVMs. As a completelynon-invasive method, 4D dMRA offers hemodynamic information with atemporal resolution of 50-100 ms for the evaluation of AVMs and cancomplement existing methods such as DSA and TOF MRA.In our study out of 32 patients, 10(31.25\%) patients are havingCerebral Aneurysms. $60 \%$ of aneurysms are seen in females and $40 \%$ observed in males and females are more susceptible than men. ${ }^{[12]}$ Females are more prone toaneurysm rupture, with SAH 1.6 times more common in women. Aneurysmsalso run in families in the absence of an identified genetic disorder, with aprevalence of $7 \%$ to $20 \%$ in first- or second-degree relatives of patients whohave suffered a SAH. ${ }^{[3,14]}$ Juan R. Cebrald et al; ${ }^{[15]}$ In their Report Stated that Hemodynamicfactors are thought to play an important role in the initiation, growth, andrupture of cerebral aneurysms.In this study, $40 \%$ of aneurysms are seen in the 4 th decade and $30 \%$ of aneurysms are observedin the 5th decade, which correlates with the literature.In our study, out of 10 aneurysms, $40 \%$ of aneurysms areobserved at Acom, 30\% are at MCA and $10 \%$ of each occurred at Pcom, ICA, and A2 segment of ACA. $50 \%$ of aneurysms had an average size of 6-7 mmand $20 \%$ of aneurysms had an average size of 7-8 mm.Young-Gyun Jeong et al; ${ }^{[16]}$ Conducted a study in 336 patients withaneurysms to determine whether there is a critical aneurysm size at which theincidence of rupture increases and whether there is a correlation betweenaneurysm size and location. They concluded that Ruptured aneurysms in theACA were smaller than those in the MCA.Hemorrhage. In our study $60 \%$ of patients presented with SubarachnoidHaemorrhage and $40 \%$ presented with headache.In our Study, TOF MRA able to diagnose all 10 aneurysms. However, in most of the cases, it failed to show the characteristics of theaneurysms like exact size and lobulations and origin of branch vessel arisingfrom the intracranial aneurysmal sac and failed to demonstrate the saccontents.

\section{Conclusion}

MRA is now in routine use as a non-invasive tool for imaging the cerebralvasculature. In cerebrovascular disease, it is the investigation of choice forpatients who are suspected/ increased risk of having unruptured intracranialaneurysms, and intracranial vascular disease associated with acute infarction, intracranial dissection of the carotid and/or vertebral arteries, and follow upcases of Cerebral AVMs. It has got some limitations in detecting smallaneurysms $(<3 \mathrm{~mm})$ and aneurysmal sac contents in case of giant aneurysmsand venous drainage patterns in case of Cerebral AVMs and to show thecomplete nidus obliteration in follow up cases of cerebral
AVM's. So, MRAshould not be the sole imaging technique in all the above conditions it should besupplemented with novel MRA techniques like CE-MRA, Time Resolving MRA, and MR DSA for adequate detection and characterization of lesions.

\section{REFERENCES}

1. RR Edelman. MR angiography: present and future. AJR Am J Roentgenol 1991;161(1):1-11.

2. May Nour, David S Liebeskind. Imaging of Cerebral Ischemia: From Acute Stroke to Chronic Disorders. Neurol Clin. 2014; 32(1).

3. Vu D., González R.G., Schaefer P.W. Conventional MRI and MR Angiography of Stroke. Acute Ischemic stroke. Springer-Verlag Berlin Heidelberg 2006;115-137.

4. JS Ross, TJ Masaryk, MT Modic, PM Ruggieri et al. Intracranial aneurysms: evaluation by MR angiography. Am J Neuroradiol 1990; 11(3):449-55.

5. Vijay Y, Sameer AS, BP Walcott, BR Buchbinder et al. Noncontrast $3 D$ time-of-flight magnetic resonance angiography for visualization of intracranial aneurysms in patients with absolute contraindications to $\mathrm{CT}$ or MRI contrast. J Clin Neurosci 2013; 20(8):1122-26.

6. The Arteriovenous Malformation Study Group. Arteriovenousmalformations of the brain in adults. N Engl J Med 1999;340:1812-18.

7. Padget DH. Cranial venous system in man about development, adult configuration, and relation to arteries. Am J Anat 1956;98(3):307-55.

8. De Biase L, Di Lisi F, Perna S, Spallone A, Ferranti F, Lucani $A$, et al. Recurrent episodes of syncope in a patient with cerebral arteriovenousmalformation. Clin Ter 2007;158(2):147-50.

9. Saleh RS, Lohan DG, Villablanca JP, Duckwiler G, Kee ST, Finn JP.Assessment of Craniospinal Arteriovenous Malformations at 3T with HighlyTemporally and highly Spatially Resolved Contrast-Enhanced MRAngiography. AJNR Am J Neuroradiol 2008; 29(5):1024-31.

10. Scott W, Atlas. Magnetic Resonance Imaging of Brain and Spine. 4th ed.Philadelphia, Pa: Lippincott, Williams \&Wilkins; 2008; 698-708.

11. Yu S, Yan L, Yao Y, Wang S, Yang $M$, Wang $B$, et al. Noncontrastdynamic MRA in intracranial arteriovenous malformation (AVM), comparisonwith the time of flight (TOF) and digital subtraction angiography (DSA). MagnReson Imaging 2012;30(6):869-77.

12. Linn FH, Rinkel GJ, Algra A, van Gijn J. Incidence of subarachnoidhemorrhage: role of region, year, and rate of computed tomography: a meta-analysis. Stroke1996;27(4):625-29. 
13. Gasparotti R, Liserre R. Intracranial aneurysms. Eur Radiol 2005;15:441-447.

14. Wardlaw JM, White PM. The detection and management of unrupturedintracranial aneurysms. Brain 2000;123(2):205-21.

15. Juan R. Cebral, Marcelo A. Castro, Burgess JE, Pergolizzi RS, SheridanMJ, Putman CM. Characterization of Cerebral Aneurysms for Assessing Risk of Rupture by Using PatientSpecific Computational Hemodynamics Models.AJNR Am J Neuroradiol 2005;26(10):2550-59.

16. Young-Gyun Jeong, Yong-Tae Jung, Moo-Seong Kim, Choong-Ki Eun,Sang-Hwan Jang. Size and Location of Ruptured Intracranial Aneurysms. JKorean Neuro Surg Soc 2009;45(1):11-15.

How to cite this article : Srinivas V, Sai Venkata Rammohan. Role of Magnetic Resonance Angiography in Evaluation of Brain Pathologies.Perspectives in Medical Research 2021; 9 (2): 77-81

DOI:10.47799/pimr.0902.17

Sources of Support: Nil, Conflict of interest: None declared 